\title{
Description of the Enzymatic Browning in Avocado Slice Using GLCM Image Texture
}

\author{
Roberto Quevedo ${ }^{1, *}$, Emir Valencia ${ }^{1}$, José Miguel Bastías ${ }^{2}$, and Stefany Cárdenas ${ }^{3}$ \\ ${ }^{1}$ Departamento de Acuicultura \& Recursos Agroalimentarios \\ Universidad de Los Lagos, Osorno, Chile \\ rquevedo@ulagos.cl \\ ${ }^{2}$ Departamento de Ingeniería en Alimentos, Universidad del BioBio, Chillán, Chile \\ ${ }^{3}$ Departamento de Ingeniería Bioquímica Escuela Nacional de Ciencias Biológicas del \\ Instituto Politécnico Nacional, México D.F.
}

\begin{abstract}
The browning kinetic was recorded using color data information from images of avocado slices. GLCM image texture was used to describe the reaction. In the experiment, images of avocado slices stored at $4^{\circ} \mathrm{C}$ were captured and saved in tiff format. The classical color intensity index (the mean $\mathrm{L}^{*}$ value) and some statistics GLCM image textures were used. Results showed that it is possible to use GLCM image texture to model the browning kinetic, because the surface intensity in one image becomes more jagged and local variations in color intensity are distributed non-homogeneously on the image during browning. The rate derived from the mean $\mathrm{L}^{*}$ color intensity was similar to those derived from the energy texture; but in general, excepted for the Energy texture index, rates generated using the texture images produce different values from those obtained using the classical browning index.
\end{abstract}

Keywords: GLCM, image texture, avocado.

\section{Introduction}

The texture of images, also called texture feature (TF), is a set of metrics calculated in image processing used to quantify the perceived texture of an image. Image Texture gives us information about the spatial arrangement of color or intensities in an image or selected region of an image [1]. It is an important tool used in pattern recognition to characterize the arrangement of basic constituents of a material on a surface [2].

Texture of images reflects changes of intensity values of pixels, which might contain information about the geometric structure of objects: a large change in intensity values often indicates a change in geometric structure. Image Texture is also an important type of information used by humans when analyzing scenes and an innate property of all surfaces.

Texture is an important image feature for describing properties of objects in images and it has been introduced into a wide range of application such as metal surface analysis, characterization of textiles, cell recognition and counting, ultrasonic images processing and food quality evaluation [3,4]. Texture of images can be classified [5]

\footnotetext{
* Corresponding author.
} 
into four groups: statistical texture, structural texture, model based texture, and transform-based texture. In the food industry, statistical texture and the transform-based texture has been the most widely used methods for quality grading or classification. The grey level co-occurrence matrix is one of the mostly used statistical texture analysis method, in which TF is extracted from the co-occurrence matrix by some statistical approaches. The co-occurrence matrix captures numerical features of a texture using spatial relations of similar gray tones [6]. Numerical features computed from the co-occurrence matrix can be used to represent, compare, and classify textures. In recently years, the transform-based texture (specifically Fourier Texture image) has been used not only to represent, compare, and classify textures; it has also been used to describe reaction kinetics in fruit and vegetables [7-9]. However, the potential of the co-occurrence matrix method to describe a kinetic reaction in food has not yet been demonstrated.

In this context, the co-occurrence matrix method, such as Contrast (con), Energy $(E)$, Homogeneity $(H)$ and - may be the most classic image texture - the standard deviation of the data on the images, can be used in order to describe reaction kinetics in food. The aim of this work is to apply the above-mentioned textural methods in order to determine whether they can be used as indicators to describe reaction kinetics in food, specifically in avocado.

\section{Materials and Methods}

\subsection{Image Samples}

Avocado images, corresponding to unpublished data from Quevedo et al [10] were used. Twelve slices each $10 \mathrm{~mm}$ thick and each placed into a $2 \mathrm{~cm}$ x $2 \mathrm{~cm}$ container were used as samples. They were placed together under the camera of a computer vision system (CVS). Pictures were taken every five minutes during 750 minutes. The experiments were replicated three times at a room temperature of $4^{\circ} \mathrm{C}$. The photographed images (2800 x 2100 pixels) were stored in Tiff format.

The samples were illuminated using four fluorescent lamps TL-D deluxe, natural daylight and 18 W/965 (Philips, Santiago, Chile) with a color temperature of $6500 \mathrm{~K}$ (D65, standard light source commonly used in food research) and a colour-rendering index ( $\mathrm{Ra}$ ) close to $95 \%$. Lamps (60 cm long) were arranged in the form of a square, $35 \mathrm{~cm}$ above the sample and at an angle of $45^{\circ}$ in relation with the sample. Additionally, light diffusers covering each fluorescent lamp and electronic ballasts ensured a uniform illumination system.

\subsection{Image Texture Analysis}

Color images were transformed to $\mathrm{L}^{*} \mathrm{a} * \mathrm{~b} *$ color space using the quadratic model function proposed by Leon et al. [11]; this transformation considers the influence of the square of the variables $(R, G, B)$ on the estimate of the values of $L^{*} a^{*} b^{*}$. The well-known textural parameter called the Gray-Level Co-Occurrence Matrix (GLCM function) was applied to the images. They characterize the texture of an image by 
calculating how often pairs of pixels with specific values and in a specified spatial relationship occur in an image [6]. The GLCM function from Matlab® R2010a (The MathWorks company) was used to compute these textural features: Contrast (con) measures the local variations in the level co-occurrence matrix. In this equation, $P$ is the number of co-appearances of red levels $i$ and $j$ ( $i$ and $j$ are two different red levels of the image). $D$ is the maximum number of pixels at a given distance and $n$ is the next pixel. As per Tan[12], only the direction of zero degrees (horizontal) and a distance of $D=3$ were used:

$$
\text { con }=\sum_{n=0}^{D} n^{2}\left(\sum_{i} \sum_{\substack{j \\|i-j|=n}} P(i, j)\right)
$$

Homogeneity or Inverse difference moment was calculated according to:

$$
H=\sum_{i, j}^{N-1} \frac{P(i, j)}{1+(i-j)^{2}}
$$

Energy, is the sum of squared elements in the GLCM:

$$
E=\sum_{i, j} P(i, j)^{2}
$$

Additionally, the classic image texture analysis based on the standard deviation $(S D)$ of the data on the images (sdtfilt function in Matlab® R2010a) was applied. Statistical parameters can characterize the texture of an image because they provide information about the local variability of the intensity values of pixels in an image [13].

\subsection{Kinetic Model of Oxidation}

In order to express a kinetic rate of browning, the extensional power law model [14] was applied to the data:

$$
\frac{\mathrm{C}_{\mathrm{t}}}{\mathrm{C}_{0}}=\exp \left(\mathrm{K} \cdot \mathrm{t}^{\mathrm{n}}\right)
$$


Where $\mathrm{C}_{\mathrm{t}}$ is the average of the $\mathrm{L}^{*}$ values on the image; or the image texture value at time $t$ respectively; $\mathrm{C}_{0}$ is the initial value, $\mathrm{K}$ is a rate parameter, and $\mathrm{n}$ is a parameter called "shape factor". The shape factor, in Equation 4, is a measure of the degree of concavity and the direction of the fitted curve [15]. Data were fitted to the model by the least-squares method, using the "solver" function, from Excel 2010 (Microsoft® Company). ANOVA and the LSD test (95\% accuracy) were used in order to compare the averaged kinetic rates and to detect statistical differences between methods. The ANOVA function in Excel 2010 (Microsoft ${ }^{\circledR}$ Company) was used.

\section{$3 \quad$ Results and Discussions}

In the Figure 1, a gallery of four selected images (taken at different times in the experiment) corresponding to one avocado sample, and their corresponding surface intensity (based on $\mathrm{L}^{*}$ value) are showed. The surface intensity becomes more jagged or complex when the reaction occur $[8,10,16]$ due to changes in the local variations on the image. In Figure 2, the browning kinetic expressed using the mean $\mathrm{L}^{*}$ value corresponding to the sample in Figure 1. The mean $\mathrm{L}^{*}$ value (luminance) decreased during the browning kinetic because the sample becomes dark over time[17]. Values can be fitted to the proposed model (Equation 1) in order to obtain the rate $(\mathrm{K})$.

In Figures 3 to 6, show the kinetics using the image textures, corresponding to the sample in Figure 1. As can be expected, these texture values increased or decreased monotonically over time, indicating changes in the color intensity values on the images. It is an indication that colors are distributed non-homogeneously on the surface. In the case of the con texture, values increased from the 2.7 to 8.7 , indicating that changes in the local variations of the $\mathrm{L}^{*}$ value (at distance of 3 pixels) increased the browning (Figure 3). With respect to the Energy $(E)$, it decreased from 0.043 to 0.014 , indicating non-homogeneous changes in the energy value on the image. The value $\mathrm{H}$ Homogeneity value $(H$, Figure 5$)$, which measures local changes in image texture, decreased from 0.61 to 0.47 during browning. Finally, SD values (Figure 6) increased from 2.24 to 3.80 showing that the data are dispersed heterogeneous on the image during browning. Enzymatic browning has been recognized as a first order kinetic (n =1); $E$ and $H$ were the GLCM features with values near to 1 .

Kinetic rates can be obtained by fitting the texture data to the Equation 1, for each avocado samples; the averages and their standard deviations are reported in the Table 1. No statistical difference was detected between the rate calculated using the mean $\mathrm{L}^{*}$ and that calculated by the $E$; However, statistical differences were detected between the other rates. These results shown that it is possible to use GLCM texture analysis in order to describe the browning kinetic in avocados, because they increased or decreased monotonically with the time, at least during the time period of the experiment. 

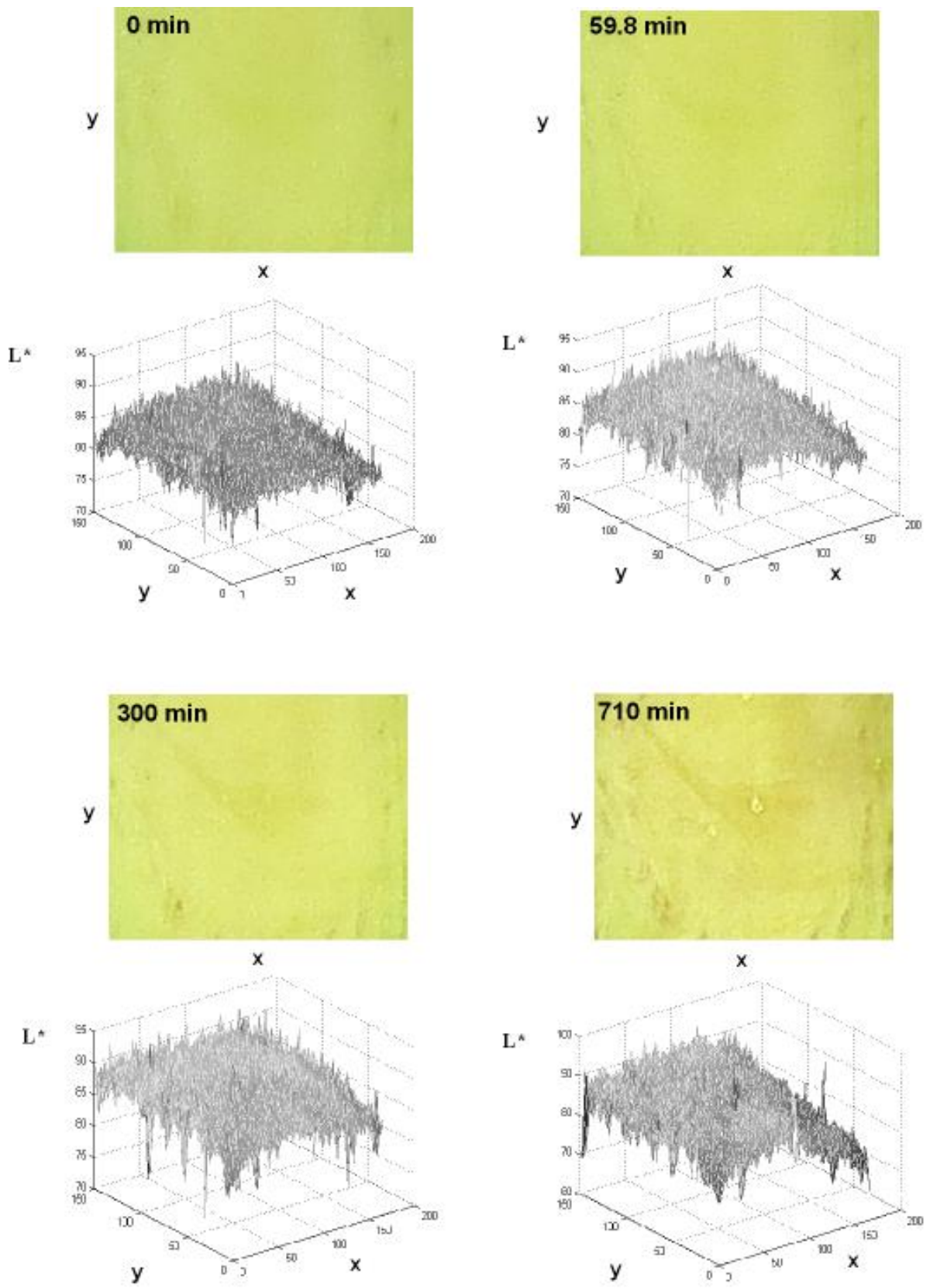

Fig. 1. Image of the one sample (avocado surface) during the kinetic at $4{ }^{\circ} \mathrm{C}$ 


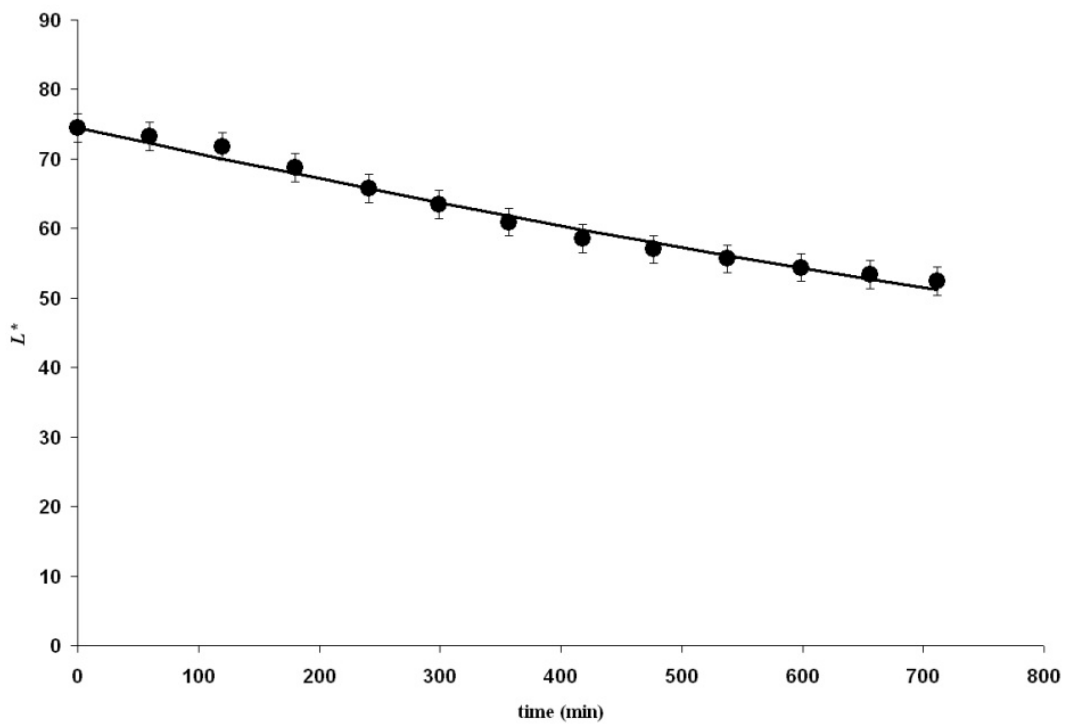

Fig. 2. Change in the mean $L^{*}$ value during the kinetic; corresponding to sample in Figure 1

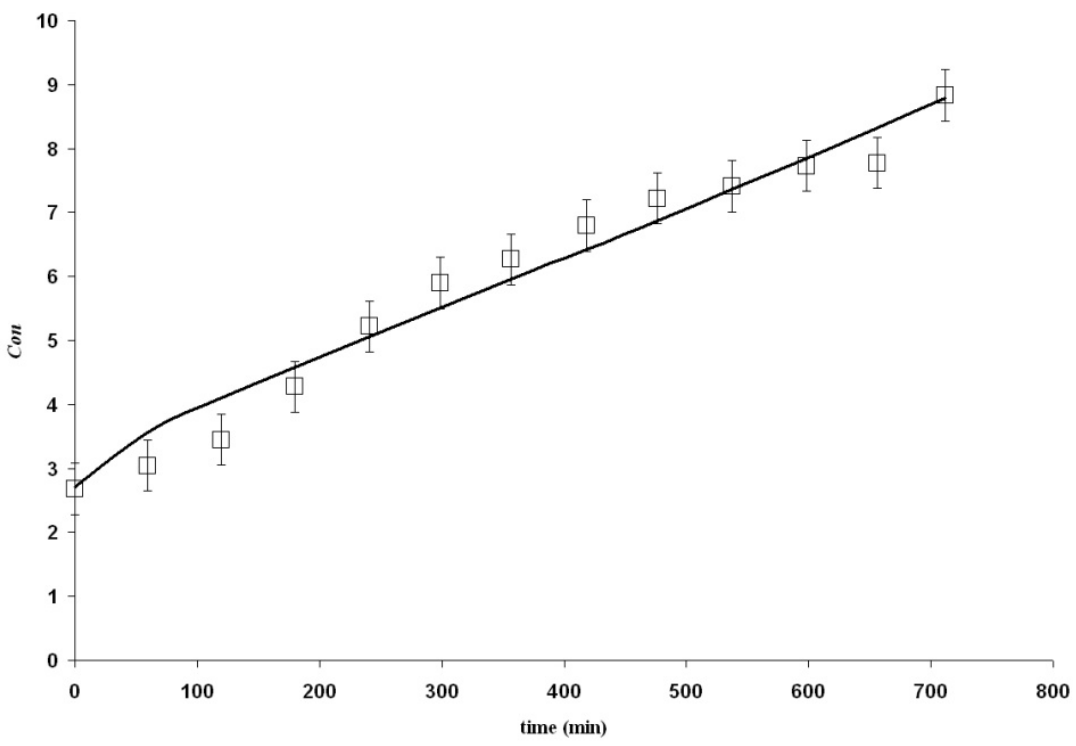

Fig. 3. Change in the image texture (con) value during the kinetic; corresponding to sample in Figure 1 


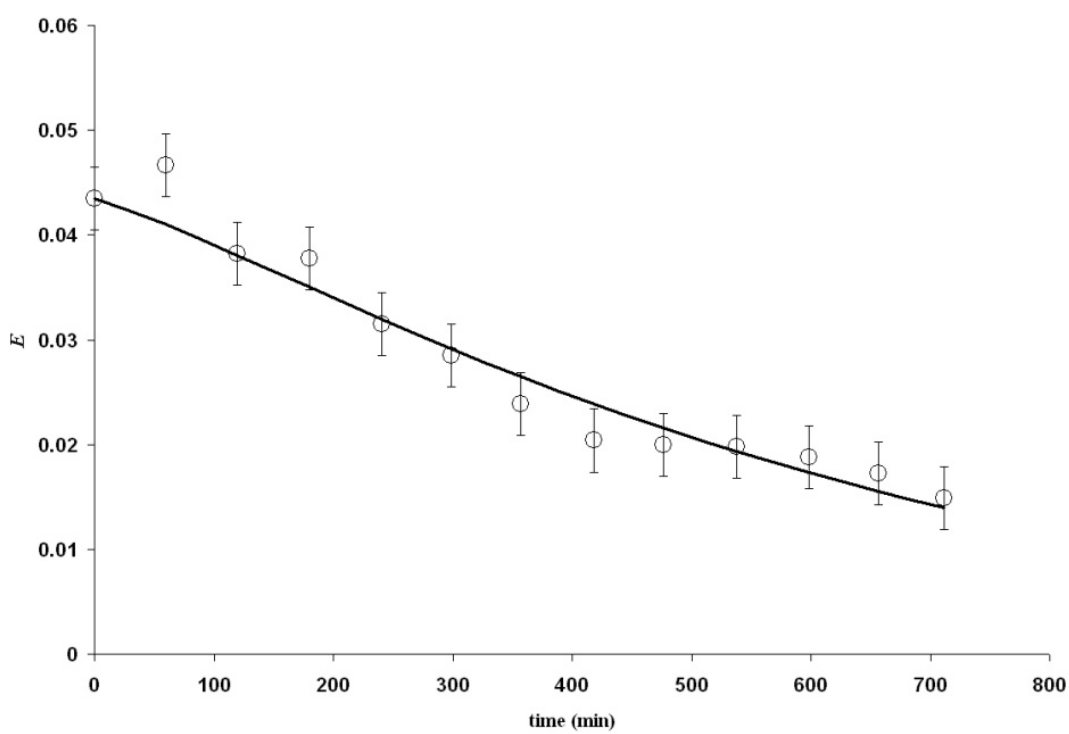

Fig. 4. Change in the image texture $(E)$ value during the kinetic; corresponding to sample in Figure 1

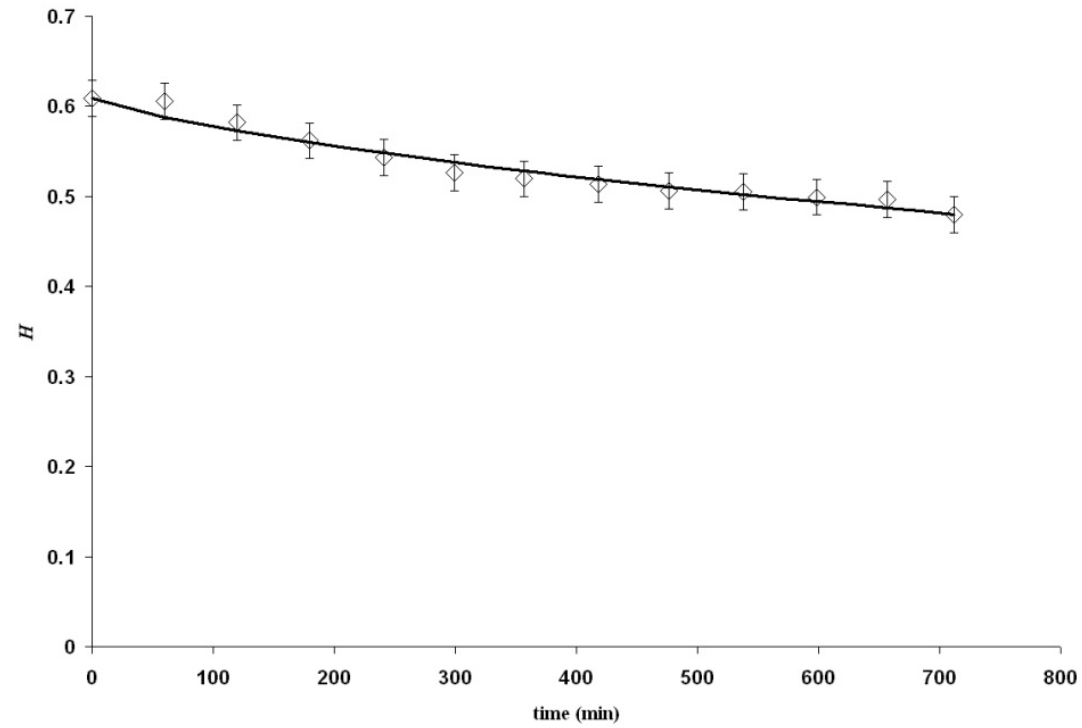

Fig. 5. Change in the image texture $(H)$ value during the kinetic; corresponding to sample in Figure 1 


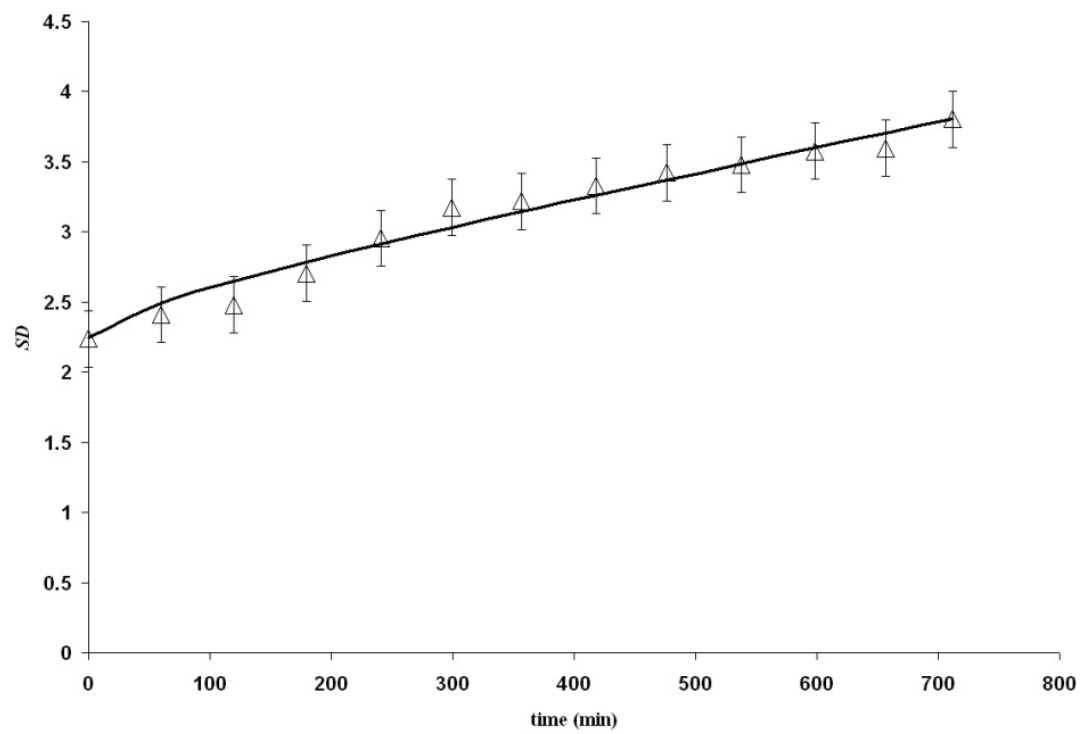

Fig. 6. Change in the image texture $(S D)$ value during the kinetic; corresponding to sample in Figure 1

Table 1. Kinetic Rate derived using differents browning index

\begin{tabular}{llll}
\hline Browning índex & Rate $\left(\mathrm{min}^{-1}\right)$ & n value & r coefficient \\
\hline Mean L* & $-0.00048 \pm 0.0001^{\mathrm{a}}$ & $1.01 \pm 0.1$ & $0.993 \pm 0.005$ \\
Con & $0.02718 \pm 0.03^{\mathrm{b}}$ & $0.57 \pm 0.25$ & $0.984 \pm 0.030$ \\
$E$ & $-0.00042 \pm 0.0004^{\mathrm{a}}$ & $1.20 \pm 0.3$ & $0.976 \pm 0.021$ \\
$H$ & $-0.00162 \pm 0.02^{\mathrm{c}}$ & $0.76 \pm 0.4$ & $0.980 \pm 0.035$ \\
$S D$ & $0.00754 \pm 0.003^{\mathrm{d}}$ & $0.64 \pm 0.4$ & $0.986 \pm 0.029$ \\
\hline
\end{tabular}

\section{Conclusions}

A browning kinetic was recorded using color data information from images of avocado slices using the GLCM image texture in order to describe the browning oxidation reaction and to derive a browning rate. Results showed that it is possible to use GLCM image textures to model browning kinetic; because intensity surface (in one image) becomes more jagged and that the local variation in color intensity changes non-homogeneously during the browning. In other word, considering that the enzymatic browning is heterogeneous in essence, GLCM image textures can describe the reaction without using the average color data on images. The rate derived from the mean $\mathrm{L}^{*}$ intensity color was similar to that derived from the energy texture; but in general, excepted the energy texture index, rates generated using the image textures give different values from those obtained using the classical browning index. 
Acknowledgment. This research was supported by the project FONDECYT-Chile number 1130745.

\section{References}

1. Zheng, C., Sun, D., Zheng, L.: Recent developments and applications of image features for food quality evaluation and inspection - a review. Trends in Food Science \& Technology $17(12), 642-655$ (2006)

2. Amadasun, M., King, R.: Textural Features Corresponding to Textural Properties. IEEE Transactions on Systems Man and Cybernetics 19(5), 1264-1274 (1989)

3. Chan, K.L.: Quantitative Characterization of Electron Micrograph Image Using Fractal Feature. IEEE Transactions on Biomedical Engineering 42(10), 1033-1037 (1995)

4. Chen, D.R., et al.: Classification of breast ultrasound images using fractal feature. Clinical Imaging 29(4), 235-245 (2005)

5. Bharati, M.H., Liu, J.J., MacGregor, J.F.: Image texture analysis: methods and comparisons. Chemometrics and Intelligent Laboratory Systems 72(1), 57-71 (2004)

6. Haralick, R., Shanmugam, K., Its'Hak, D.: Textural Features for Image Classification. IEEE Transactions on Systems, Man and Cybernetics 3 (1973)

7. Quevedo, R., et al.: Quantification of enzymatic browning kinetics in pear slices using non-homogenous L* color information from digital images. LWT - Food Science and Technology 42, 1367-1373 (2009)

8. Quevedo, R., et al.: Description of the kinetic enzymatic browning in banana (Musa cavendish) slices using non-uniform color information from digital images. Food Research International 42(9), 1309-1314 (2009)

9. Quevedo, R., et al.: Quantification of enzymatic browning in apple slices applying the fractal texture Fourier image. Journal of Food Engineering 95(2), 285-290 (2009)

10. Quevedo, R., et al.: Enzymatic browning in sliced and puréed avocado: A fractal kinetic study. Journal of Food Engineering 105(2), 210-215 (2011)

11. Leon, K., et al.: Color measurement in $\mathrm{L}^{*} \mathrm{a} \mathrm{b}^{*}$ units from RGB digital images. Food Research International 39, 1084-1091 (2006)

12. Tan, J.: Meat quality evaluation by computer vision. Journal of Food Engineering 61, 27-35 (2004)

13. Basset, O., et al.: Application of texture image analysis for the classification of bovine meat. Food Chemistry 69(4), 437-445 (2000)

14. Peleg, M., Corradini, M.G., Normand, M.D.: Kinetic models of complex biochemical reactions and biological processes. Chemie Ingenieur Technik 76(4), 413-423 (2004)

15. Peleg, M., Normand, M., Corradini, M.: The Arrhenius equation revisited. Critical Review Food Science and Nutrition 52(9), 830-851 (2012)

16. Quevedo, R., et al.: Comparison of Whiteness Index vs. Fractal Fourier in the Determination of Bloom Chocolate Using Image Analysis. Food Bioprocess Technol. (2011), doi:10.1007/s11947-011-0729-x

17. Luo, Y., Barbosa, G.: Enzymatic browning and its inhibition in new apple cultivars slices using 4-hexylresorcinol in combination with ascorbic acid. Food Science and Technology International 3(3), 195-201 (1997) 